Revista Signos 2009, 42(71) 295-315

\title{
Estrategias de expresión de la evidencialidad en la argumentación oral en sala de clases*
}

\author{
Carlos González \\ Pablo Lima \\ Pontificia Universidad Católica de Chile \\ Chile
}

\begin{abstract}
Resumen: Este trabajo tiene como objetivo identificar la presencia de estrategias gramaticales de expresión del significado evidencial en un corpus de cinco discusiones argumentativas orales de alumnos de tercer año medio de un colegio de Viña del Mar. El modelo utilizado tanto para la búsqueda de fenómenos como para su interpretación se fundamenta esencialmente en las propuestas de Bermúdez (2002, 2005a, 2005b). El análisis de las clases muestra que los alumnos sí utilizan marcas evidenciales en su interacción, aunque en un número escaso. La estrategia evidencial más destacada es el uso del pronombre 'uno' y su relación paradigmática con 'yo' y frases nominales genéricas, estrategia que transmite significados evidenciales relacionados con los ejes de 'fuente de información' y 'acceso a la información'. La presencia de evidenciales parece ser favorecida por la cercanía de los estudiantes con el tema de discusión y por un bajo grado de estructuración de la interacción.
\end{abstract}

Palabras Clave: Evidencialidad, estrategias evidenciales, argumentación oral.

* Proyectos FONDECYT 11070002 Formas gramaticales de expresión de la evidencia en el español de Chile y FONDECYT 1060439 La competencia argumentativa oral en el aula: Un estudio exploratorio con estudiantes de enseñanza media.

Recibido:

3-III-2008

Aceptado: 30-IV-2009
Correspondencia: Carlos González (cgonzalv@uc.cl). Departamento de Ciencias del Lenguaje, Pontificia Universidad Católica de Chile. Avenida Vicuña Mackenna 4860, Santiago, Chile. 


\title{
Strategies to express evidentiality in oral argumentation within the classroom
}

\begin{abstract}
This article identifies the presence of grammatical strategies to express the evidential meaning in a corpus of five oral argumentative discussions in Viña del Mar high school students. Bermúdez's (2002, 2005a, 2005b) model for the search of phenomena as well as for their interpretation was used. The analysis shows that the students do use evidentials in their interactions, but only in a few cases. The most common strategy is the use of the pronoun uno and its paradigmatic relation with yo and generic nominal phrases. This strategy conveys evidential meaning related to two axes: 'source of information' and 'access to information'. The analysis also reveals that the number of evidentials used by the students seems to be directly related to the knowledge of the issue and inversely related to a low degree of organization of the discussion.
\end{abstract}

Key Words: Evidentiality, evidentiality strategies, oral argumentation.

\section{INTRODUCCIÓN}

En las discusiones cotidianas, uno de los aspectos más importantes que el hablante puede expresar en cada aseveración es la información sobre la fuente en que se basa para emitir sus juicios. Es habitual que en español esta referencia se materialice en frases o elementos léxicos independientes como 'como dice este autor...', 'todo el mundo sabe que...' o 'evidentemente', por citar algunos ejemplos; sin embargo, existen lenguas que poseen también medios gramaticales sistemáticos para señalar este significado, entre ellas, lenguas indígenas americanas como el quechua o el tariana ${ }^{1}$. El dominio semántico concerniente a la indicación de la fuente de información es conocido como 'evidencialidad' y las formas de expresarlo por medios gramaticales, 'marcadores' o 'estrategias evidenciales'.

En opinión de autores como Aikhenvald (2004), la expresión gramatical sistemática de la evidencialidad en marcadores específicos no es un fenómeno que se produzca en las lenguas mejor conocidas, como el inglés, el francés o el español. Sin embargo, investigaciones recientes en nuestra lengua han mostrado que sí es posible considerar que elementos sistemáticos de ella, como ciertos morfemas o construcciones sintácticas, expresen significados de este dominio, a la manera de 'estrategias evidenciales' (véase el apartado 1.2. para mayores detalles). De entre estas investigaciones, destacan las propuestas de Bermúdez (2002, 2005a, 2005b).

Dada la relación existente entre evidencialidad y la referencia a la fuente de información, parece sensato pensar que las interacciones argumentativas, en las que el hablante se ve constantemente ante la exigencia de justificar sus afirmaciones, conforman un campo propicio para hallar manifestaciones gramaticales de este domino semántico. 
Como se plantea en van Eemeren, Houtlooser y Snoeck Henkemans (2007), los diferentes movimientos que forman parte de la argumentación suelen hacer uso de marcadores específicos, llamados 'indicadores argumentativos'. Específicamente, en la expresión del punto de vista propio, es frecuente que el hablante, ya sea por motivación propia o por exigencia de la contraparte, sienta la necesidad de señalar en qué fuente de información se basa para aseverar algo. Esta indicación suele realizarse por medios léxicos; sin embargo, si el español cuenta efectivamente con medios gramaticales sistemáticos relacionados con la evidencialidad, sería esperable encontrarlos justamente en la práctica discursiva de la argumentación.

A partir de esto, la pregunta que guía nuestra investigación es: ¿es posible detectar estrategias gramaticales específicas y sistemáticas que expresen significados relacionados con la fuente de información en la interacción argumentativa? y, consecuentemente, el objetivo que nos planteamos es observar la presencia de estrategias gramaticales de expresión de la evidencialidad, específicamente en un corpus consistente en la transcripción de cinco discusiones orales argumentativas producidas en el contexto de clases de Lenguaje y Comunicación de estudiantes de tercer año de enseñanza media de un colegio de Viña del Mar, cada una de aproximadamente 45 minutos de duración.

Este estudio pretende, por una parte, contribuir al conocimiento del sistema lingüístico del español en general y del español de Chile en particular por medio de la observación de estrategias gramaticales que expresen información relacionada con el dominio evidencial y, por otra parte, observar si en la interacción argumentativa ocupan un lugar destacado tales estrategias.

La organización de este artículo es la siguiente: en el apartado 1, presentamos el concepto general de evidencialidad, las propuestas existentes de estrategias de expresión de ella en español y el modelo postulado por Bermúdez (2005a), en que nos basamos para organizar nuestro estudio; en el apartado 2, precisamos el objeto de estudio y el método utilizado tanto para la recolección del corpus como para su análisis; en el apartado 3, ofrecemos la descripción de las estrategias gramaticales halladas en las discusiones, deteniéndonos especialmente en el análisis de un recurso no descrito hasta el momento en la literatura: el uso del pronombre 'uno' y su paradigma; en el punto 4, relacionamos la frecuencia de aparición de estrategias evidenciales con diferentes factores que pueden influir en ella, como el número de intervenciones, el tema de discusión y el grado de estructuración de cada clase; finalmente, en las conclusiones resumimos nuestros hallazgos y discutimos a partir de ellos la relación entre evidencialidad y la práctica discursiva de la argumentación. 


\section{Marco de referencia}

\subsection{La evidencialidad}

La evidencialidad corresponde al dominio de significado que expresa por medios lingüísticos cuál es la fuente de información en que un hablante se basa para emitir un enunciado. Estos medios, denominados generalmente 'marcadores evidenciales' o 'estrategias evidenciales', son variados y pueden comprender desde morfemas específicamente dedicados hasta construcciones sintácticas, si bien no todas estas formas de expresión son unánimemente aceptadas como válidas entre los teóricos.

Tradicionalmente, el dominio de la evidencialidad se ha asociado con lenguas 'exóticas', como las de la familia tibeto-burmana o lenguas amerindias, como el tagalo o el quechua. Por ejemplo, el tariana, una lengua de la familia arahuacana hablada en la zona noroccidental del Amazonas, se caracteriza por ser capaz de expresar por medio de un morfema verbal obligatorio cinco distintas posibilidades por las que el hablante puede haber llegado a obtener la información que expresa, como puede verse en las oraciones del ejemplo (1), en las que se marcan en negrita tanto el morfema evidencial como su significado en la glosa (Aikhenvald, 2004)².

(1) a. Juse irida di-manika-ka

José fútbol 3sg.NF-jugar-PASREC.VIS

José ha jugado fútbol (yo lo vi jugar)

b. Juse irida di-manika-mahka

José fútbol 3sg.NF-jugar-PASREC.NOVIS

José ha jugado fútbol (yo lo oí jugar)

c. Juse irida di-manika-nihka

José fútbol 3sg.NF-jugar-PASREC.INFER

José ha jugado fútbol (yo lo infiero a partir de pistas)

d. Juse irida di-manika-sika

José fútbol 3sg.NF-jugar-PASREC.PRESUN

José ha jugado fútbol (yo lo asumo a partir de lo que sé de él)

e. Juse irida di-manika-pidaka

José fútbol 3sg.NF-jugar-PASREC.REP

José ha jugado fútbol (alguien me lo contó) 
En el ejemplo (1a), el hablante afirma que 'José ha jugado fútbol' e informa que la fuente en que se basa para decir esto es su percepción visual directa del hecho; en (1b), expresa que la misma afirmación está respaldada no por la observación sino por otro medio de acceso sensorial, como el oído, por ejemplo; en el caso (1c), la aseveración se establece a partir de la percepción de evidencia visual que lleva al hablante a inferir lo que ha pasado (en este caso, podría tratarse de la observación de que las zapatillas de José están sucias); en (1d), el hablante establece su afirmación fundamentándola en lo que él sabe de la conducta habitual de José (por ejemplo, que él siempre juega al fútbol un día determinado de la semana); finalmente, en (1e) la aseveración de que 'José ha jugado fútbol' es expresada por el hablante con la indicación de que se trata de un evento que le ha sido referido, es decir, se trata de información de segunda mano. El del tariana es uno de los sistemas de expresión de la evidencialidad más complejos de los que se tenga registro (Aikhenvald, 2004) y, como se puede observar en los ejemplos citados, sus diferentes significados se materializan en morfemas verbales específicos, si bien amalgamados con el significado temporal.

Una forma distinta de expresión de la evidencialidad es la que se presenta en boumaa, un dialecto del fijiano estándar (Dixon, 1988). En esta lengua, la presencia o ausencia de un complementante en la cláusula subordinada de una oración como la del ejemplo (2) señala diferencias relativas a la fuente de información: en (2a) se indica que el evento 'esos jóvenes ganaron' fue oído directamente por el hablante, mientras que en $(2 \mathrm{~b})$ la presencia de la partícula 'ni' (marcada en negrita) señala que la información fue adquirida de segunda mano. Un ejemplo como este resulta interesante, porque muestra que la evidencialidad no solo puede manifestarse por medios morfológicos, sino también mediante diferencias en la construcción sintáctica.

a.

Au aa rogo-ca [a o-dra qaaqaa a cauravou yai]

1sg PAS oír-TR ART CLASIF-3pl ganar ART jóvenes estos

Oí que esos jóvenes ganaron (oí el evento mismo)

b. Au aa rogo-ca [ni+ra qaaqaa a cauravou yai]

1sg PAS oír-TR QUE+3PL CLASIF-3pl ganar ART jóvenes estos

Oí que esos jóvenes ganaron (alguien me lo dijo)

\subsection{Formas gramaticales de expresión de la evidencialidad en español}

El hecho de que la expresión del significado relacionado con la fuente de información no se indique obligatoriamente por medio de marcadores morfemáticos especializados en las lenguas indoeuropeas ha supuesto una dificultad para que el dominio semántico de la evidencialidad reciba el mismo tratamiento que otros más conocidos, como la modalidad, el tiempo o el aspecto (De Haan, 1999; Lazard, 1999, 2001; DeLancey, 2001), tanto en lo que respecta a su especifici- 
dad de ámbito, pues es muy frecuente que la evidencialidad se considere como un subdominio o un tipo especial de modalidad epistémica (Chafe \& Nichols, 1986), como en la aceptación de que este significado pueda manifestarse por medios diferentes a los de un paradigma morfológico exclusivo.

Investigaciones recientes, sin embargo, han propuesto que las lenguas indoeuropeas, y entre ellas el español, sí expresan de manera sistemática el significado relacionado con la fuente de información, aunque este no se manifieste en forma de una marca individual y única; se trataría, en consecuencia de estrategias gramaticales de expresión del significado evidencial. Uno de los casos más conocidos de forma de expresión de la evidencia y que se encuentra frecuentemente señalado como un 'error gramatical' en los libros de estilo es el llamado 'condicional de rumor', que puede verse ejemplificado en (3). En estos casos, resulta claro que la forma 'condicional' no expresa significados temporales (pospretérito y antepospretérito, respectivamente), ni tampoco aparece en la apódosis de una estructura condicional, sino que su valor es el de señalar que lo que se comunica es información de segunda mano; en (3b), incluso, esta fuente externa es señalada explícitamente: 'Ferrocarriles'.

(3) a. Licencias Médicas: aplicación de decreto de Büchi sería permanente (La Época, 11/9/1997)

b. Disco "Pare" no habría sido respetado, según Ferrocarriles (El Mercurio, $8 / 3 / 2004)$

Bermúdez (2005b) analiza otros ‘tiempos' verbales que también son usados con valor evidencial, algunos de los cuales pueden verse en (4) (Bermúdez, 2005b). Según esta propuesta, los 'futuros' de (4a) y (4b) indican que la afirmación del hablante se basa en una inferencia, mientras que los 'pretéritos imperfectos' de (4c) y (4d) señalan que la información por la que se pregunta o que se afirma se basa en un conocimiento compartido.

(4) a. ¿Qué le pasa a Mario que no quiere bailar?

Estará cansado

b. $\quad$ No encuentro la bandeja de plata.

Se habrá perdido en la mudanza

c. ¿A qué hora era la reunión de mañana?

d. $\quad$ El tren salía a las 15.45

Bermúdez (2002) propone que la construcción española con verbos de percepción y cláusula subordinada, como la de los ejemplos de (5), manifiesta también una diferencia esencial en relación con la fuente de información. Así, en el caso de (5a) la información pudo tanto haber- 
se adquirido directamente, de manera presencial, como tratarse de información de segunda mano ('alguien me lo dijo'); en el caso de (5b), sin embargo, solo la primera interpretación es posible. La diferencia entre ambas construcciones es paralela a la del ejemplo del boumaa, ya comentada.

$$
\begin{aligned}
& \text { a. Oí que los jóvenes ganaron (los oí directamente / alguien me lo dijo) } \\
& \text { b. Oí ganar a los jóvenes (los oí directamente / *alguien me lo dijo) }
\end{aligned}
$$

Schwenter (1999) y Demonte y Fernández (2005), por otra parte, han propuesto que la presencia de la preposición 'de' en construcciones dequeístas como la del ejemplo (6) actúa como marcador evidencial, aunque en la perspectiva de estos autores el significado que se le atribuye a la estructura con 'de' es el de alejamiento del hablante con respecto a lo dicho (Schwenter, 1999) o el de bajo grado de confianza del hablante con respecto a lo manifestado en el enunciado (Demonte \& Fernández Soriano, 2005; fundamentadas en Rooryck, 2001), lo que se relaciona más bien con el dominio de la modalidad epistémica.

$$
\text { Pienso de que los conozco poco }
$$

Parece posible considerar, en consecuencia, que el dominio semántico de la evidencialidad se encuentra presente en el sistema de la lengua española, si bien los medios gramaticales de expresión de este significado no se encuentran aún catalogados de manera completa.

\subsection{Un modelo de análisis para la evidencialidad}

Desde los primeros estudios del fenómeno, se han propuesto diferentes clasificaciones que intentan ordenar el ámbito de significado de la evidencialidad. Las primeras, de orientación más estructural, se basan en la oposición de los diferentes tipos de fuente de información (Wilett, 1988; Tournadre, 1996; Plungian, 2001). Una distinción primordial para estos estudios se encuentra en el contraste entre la información obtenida por medios directos e indirectos, por una parte, y la distinción entre la información proveniente del propio hablante y la mediada por los demás, por otra. Clasificaciones de este tipo han llegado a establecer un conjunto de hasta nueve categorías o subdominios de la evidencialidad.

Bermúdez (2005a) critica este tipo de propuestas, pues presentan el inconveniente propio de todo sistema de clasificación basado en categorías rígidas: no logran capturar la complejidad de los estados de cosas reales y su relación con los participantes, complejidad que un significado como el de la evidencialidad intenta justamente expresar. Este autor argumenta convincentemente que los subdominios de la evidencialidad no se describen de manera adecuada como clases cerradas, ya que los límites que hay entre ellas son difusos en la realidad. Tal es su opinión, por ejemplo, con respecto a la oposición información personal / información transmitida: 
"Mucho del conocimiento que poseemos lo hemos adquirido a partir de fuentes externas, de segunda o tercera mano, por medio de libros o enseñanzas de personas consideradas autoridades en la materia que a su vez lo aprendieron de libros o de sus propios maestros, etc. Sin embargo, esta información transmitida se integra a nuestra concepción del mundo y es en función del grado en que tal información se asimila a nuestro sistema conceptual que la consideramos propia o ajena" (Bermúdez, 2005a:10).

A partir de reflexiones como esta, Bermúdez (2005a) propone que el dominio semántico de la evidencialidad se puede describir de mejor forma como organizado en torno a tres ejes continuos: la fuente de información (en qué lugar se halla la información); el modo de acceso a la información (la forma en que esta es adquirida) y el acceso a la información (el grado en que la información se considera compartida o no). Estos continuos tienen diferentes puntos polares (personal y ajena, para la fuente de información; sensorial y cognitiva, para el modo de acceso; y privativo y universal, para el acceso a la información) y los marcadores o estrategias evidenciales pueden transmitir un significado relacionado con cualquier punto a lo largo de estos continuos o una combinación de ellos. Este modelo se esquematiza de la siguiente manera (Figura 1).

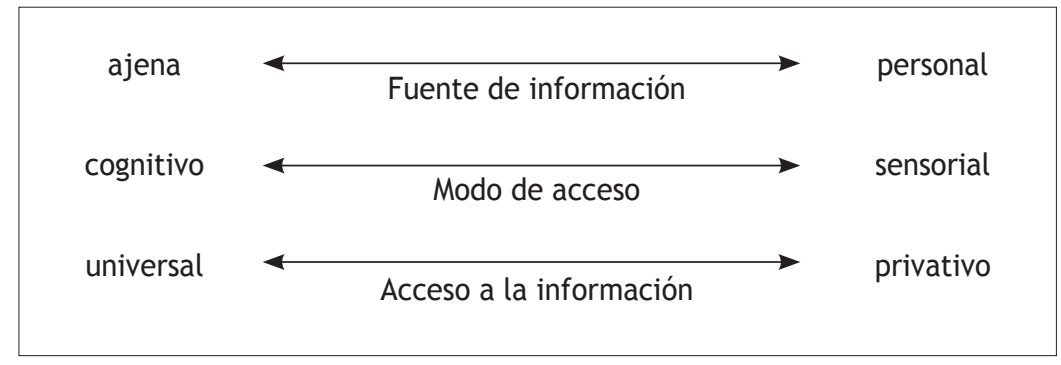

Figura 1. Continuos que describen el dominio de la evidencialidad (Bermúdez, 2005a).

Consideramos que la propuesta de Bermúdez (2005a) describe de manera más precisa y realista el complejo dominio semántico de la evidencialidad y, en consecuencia, en este estudio la utilizamos como base para nuestras propias descripciones. 


\section{Método de trabajo}

\subsection{Unidad de análisis}

Como ya se ha establecido previamente, nuestro objeto de estudio es la evidencialidad. Este será estudiado a partir de sus formas de expresión en el español de Chile. De este modo, establecemos como unidad de análisis las estrategias gramaticales que expresan significados relacionados con la fuente de información que pueden hallarse en las interacciones argumentativas en español, concretamente en las discusiones que se producen entre estudiantes de enseñanza secundaria en el contexto de clases.

\subsection{Corpus y procedimientos de análisis}

El corpus escogido está conformado por la transcripción de cinco discusiones orales producidas en el contexto de clases de la asignatura de Lenguaje y Comunicación entre estudiantes de tercer año medio de un colegio de Viña del Mar, cada una de aproximadamente 45 minutos de duración.

Las cinco sesiones analizadas, enmarcadas en el desarrollo de la unidad de argumentación, se caracterizan por presentar un tema central de controversia, generalmente introducido por el profesor, en torno al cual los alumnos entregan sus opiniones y rebaten en ocasiones los puntos de vista de los otros. Las clases tienen los siguientes tópicos centrales: clase 1: aplicación en el colegio de la jornada escolar completa; clase 2: una movilización estudiantil; clase 3: la existencia de Dios; clase 4: la clonación; clase 5: el aborto.

El procedimiento de análisis fue el siguiente: en primer lugar, se realizó la observación de cada una de las intervenciones presentes en el corpus para verificar en ellas la presencia de las estrategias evidenciales descritas por Bermúdez (2002, 2005b); es decir, el uso de tiempos verbales como los futuros simple y compuesto, el pretérito imperfecto y el condicional con valores evidenciales, así como la estructura de elevación del sujeto a objeto en verbos de percepción, como se describe en 1.2. Cada manifestación lingüística de los tipos considerados fue analizada y observada en su contexto con el fin de establecer qué ámbito semántico expresaba (así, por ejemplo, un verbo como 'era' fue, en primer lugar, identificado y clasificado como correspondiente a una de las posibles formas de expresión del significado evidencial; esta forma fue luego observada en su contexto y se estableció en consecuencia si expresaba un significado temporal, modal o, efectivamente, evidencial). Posteriormente, tratamos de identificar nuevas estrategias gramaticales que expresaran significados relacionados con el dominio evidencial, no descritas hasta el momento en la bibliografía sobre el tema. Esto se realizó mediante una nueva revisión de la totalidad de intervenciones del corpus, en la que nos centramos en la observación de las expresiones que hacían referencia, directa o indirectamente, a fuentes de información y en la 
detección de posibles estrategias sistemáticas que transmitieran ese significado. Finalmente, intentamos precisar si los temas de los que versaban las discusiones o el grado de estructuración de ellas manifestaban alguna relación con la presencia o ausencia de dichas estrategias.

\section{Estrategias evidenciales en la discusión oral en sala de clases}

\subsection{Estrategias evidenciales presentes en el corpus}

El primer punto que podemos destacar de nuestro análisis es la ausencia casi total de las estrategias de expresión de la evidencialidad descritas por Bermúdez (2002, 2005b). De hecho, de entre todas las intervenciones analizadas en las cinco clases, solamente pudimos detectar un único caso, correspondiente al uso del pretérito imperfecto con valor evidencial, el que puede apreciarse en el ejemplo (7).

\section{(7) $\quad[\ldots]$ me parece que el horario con ustedes era lunes y martes}

El enunciado de (7) corresponde a una intervención del profesor en la clase 1. Proponemos que se trata de un uso evidencial del pretérito imperfecto, ya que el contexto de la discusión da a entender que las clases de las que se describe su horario continúan impartiéndose los días lunes y martes en el momento de la discusión. En este caso, la función del imperfecto en 'era' es señalar la información como compartida entre el profesor y los alumnos con los que conversa. En términos del modelo de Bermúdez (2005a), el eje pertinente en este uso evidencial es el de acceso a la información, el que se presenta como compartido, es decir, en un punto intermedio entre el polo universal ('información disponible para todos de manera irrestricta') y el privativo ('información que posee exclusivamente el hablante').

La otra estrategia evidencial observada corresponde al uso del pronombre de tercera persona ‘uno' en casos como el del ejemplo (8), enunciado por un alumno en la discusión de la clase 3.

\section{[...] uno tiene que sufrir en esta vida para ser feliz después ${ }^{3}$}

El uso evidencial de este pronombre se vuelve claro al contrastarlo con otros elementos con los que puede alternar en el paradigma y que confieren a la expresión diferentes valores relacionados con la fuente de información, como el uso del pronombre de primera persona 'yo' o una frase nominal de interpretación genérica que incluye al hablante como 'el hombre', tal como se aprecia en los ejemplos de (9). 
a. $\quad$ yo tengo que sufrir en esta vida para ser feliz después

b. $\quad$ el hombre tiene que sufrir en esta vida para ser feliz después

La variación en la expresión de la referencia al hablante con el pronombre 'yo', el pronombre 'uno' y una frase nominal como 'el hombre' es un juego habitual en las intervenciones argumentativas de los alumnos. Proponemos que el significado evidencial de estas formas se da en su posibilidad de alternancia paradigmática y se basa en la interacción entre los ejes 'fuente de información' y 'acceso a la información'. Si el hablante elige el pronombre ‘yo', como en el ejemplo (9a), el significado que transmite es que la fuente de información es 'personal' y que el acceso a la información se considera 'privativo'; en otras palabras, indica que la información contenida en el enunciado proviene de la experiencia o creencia personal, la que no se presume necesariamente como accesible para su interlocutor. Si el hablante escoge una frase nominal (como 'el hombre' en (9b)), altera las posiciones del esquema: se vuelve neutral en cuanto a la fuente de información (no indica si es privativa o ajena), pero sí especifica que el acceso a esta información es 'universal'; es decir, el hablante señala que lo expresado en el enunciado no proviene necesariamente de su propia experiencia y que la aseveración corresponde a un hecho conocido ampliamente. Finalmente, al escoger el hablante el pronombre 'uno', señala que la fuente de información es 'personal' (al igual que en la elección de 'yo'), pero el acceso a la información se considera 'universal' (como en la elección de 'el hombre'); en otras palabras, al elegir 'uno' el hablante expresa que lo que afirma proviene de sus propias experiencias o creencias, pero que cualquiera podrá estar de acuerdo con él si atiende a las propias, como se observa en el ejemplo (8).

Este juego paradigmático puede ilustrarse en la Figura 2, que muestra los ejes pertinentes del modelo de Bermúdez y la posición en los ejes con los que se relaciona cada forma. El modelo que aquí proponemos, por supuesto, debe considerarse como una primera aproximación a la definición del significado expresado por estas formas. Su potencial explicativo estará sujeto a la confirmación de estos hallazgos en un corpus más amplio y al tratamiento estadístico de estos datos. 


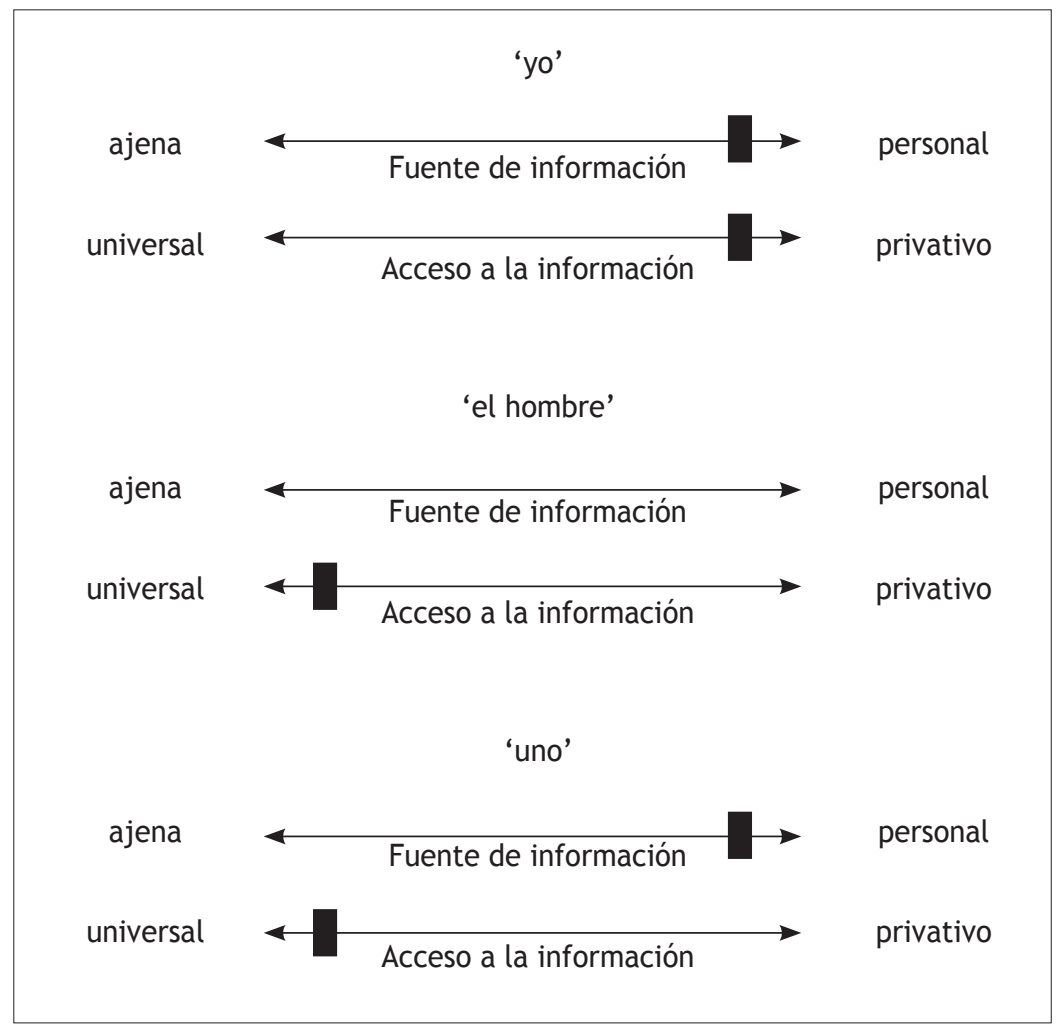

Figura 2. Posiciones en los ejes evidenciales pertinentes para el pronombre 'yo', una frase nominal genérica como 'el hombre' y el pronombre 'uno'.

\section{2. 'Uno' y su paradigma como estrategia evidencial}

El uso de 'uno' o de alguna de sus alternativas con significado relacionado con la fuente de información es la estrategia evidencial más frecuente en las discusiones analizadas, con un total de ocho apariciones. A continuación revisaremos estas, agrupadas según la clase en que se observaron. Tanto en la clase 2 (22 intervenciones), cuyo tópico central es la conveniencia de que los alumnos del colegio se plieguen a una marcha estudiantil, como en la clase 4 (46 intervenciones), cuyo tema de discusión es la clonación, no se registraron usos evidenciales de 'uno' o sus variantes. 


\subsubsection{Clase 1 (56 intervenciones)}

En esta clase, cuyo tema central es la aplicación de la jornada escolar completa en el colegio, se puede apreciar la presencia de cuatro casos relacionados con el uso evidencial de 'uno' o su paradigma. El primero es el que se expone en el ejemplo (10).

[...] el nivel de estrés es mayor, aunque no sea crítico, pero ya las personas están más estresadas y más alteradas

El ejemplo (10) corresponde a la intervención de un alumno, quien comenta cómo se han sentido él y sus compañeros con el aumento de horas de clase que ha supuesto la aplicación de la jornada escolar completa. Es por esto que la frase nominal definida 'las personas' tiene carácter genérico e incluyente en este contexto. De manera similar al ejemplo (9b), el valor evidencial de esta forma es el de fuente de información neutral y acceso a la información universal; es decir, si bien se trata de una experiencia personal, en la argumentación se presenta como si el juicio no se basara en la propia experiencia y que se trata de una opinión compartida por todos los estudiantes del colegio, que equivalen a 'las personas'. Este uso de la frase nominal definida otorga a la opinión un tono de objetividad. Algo parecido puede apreciarse en el ejemplo (11).

[...] un alumno que está desde veinte para las ocho, todo el día en el colegio y luego de un tiempo de colación que son ¿cuarenta y cinco minutos?, volver a interiorizarse en una sala y aprender después de ese tiempo, después del almuerzo, no se puede

El caso de (11) es similar al de (10). Nuevamente se trata de un estudiante que entrega su opinión sobre cómo lo ha afectado la aplicación de la jornada escolar completa (experiencia personal), pero que elige dar este testimonio utilizando una frase nominal incluyente de interpretación genérica ('un alumno'), lo que otorga a su opinión un carácter de mayor objetividad.

[...] uno llega demasiado cansado, o sea sería mucho más práctico que uno llegara a la casa y se pone a estudiar altiro

En el ejemplo (12) podemos ver el uso evidencial de 'uno'. Nuevamente se trata de un estudiante que entrega su testimonio personal sobre cómo lo ha afectado la aplicación de la jornada escolar completa. El uso del pronombre 'uno' entrega el significado de que la fuente de información es personal, pero el acceso a la información es universal; en otras palabras, el alumno enuncia que se basa en su propia experiencia para emitir el juicio, pero al mismo tiempo señala que esa misma experiencia es compartida por sus interlocutores, en este caso el resto de sus 
compañeros. Si se compara esta estrategia con las alternativas posibles: "yo llego demasiado cansado...' y 'el alumno llega demasiado cansado...', se aprecia que, con respecto a la primera, comparte la fuente personal, pero tiene la ventaja de presentar la experiencia como universalizable y, con respecto a la segunda, aunque comparte el acceso universal, el hecho de incluir como factor su propia experiencia vuelve la opinión menos distante. Parece ser entonces que el uso de 'uno' frente a sus alternativas paradigmáticas tiene ventajas retóricas.

[...] cuando salíamos a la una y media, yo llegaba a mi casa, almorzaba, dormía hasta las cuatro y a las cuatro me ponía a estudiar hasta las nueve y pasaba bien, no tenía problemas. En cambio, uno ahora llega, se sienta a ver tele un rato y ya son las seis de la tarde, las siete de la tarde y listo, se fue la tarde y vino en nada

El ejemplo (13) es interesante porque presenta una alternancia de los pronombres de primera persona 'yo' y de tercera persona 'uno'. La primera parte del enunciado se presenta como experiencia personal, accesible únicamente para el hablante. La segunda parte del enunciado, sin embargo, manifiesta un cambio de forma pronominal de 'yo' a 'uno' y, consecuentemente, lo que era una experiencia inaccesible para los interlocutores, se vuelve universal y compartida. Esta segunda parte es justamente la que critica la aplicación de la jornada escolar completa y la que presenta más fuerza argumentativa, debido al cambio de orientación de lo individual a lo general que se presenta en la intervención.

\subsubsection{Clase 3 (116 intervenciones)}

En la clase 3, cuya discusión gira en torno al tema de la existencia de Dios y la necesidad de esta existencia para el hombre, se presentan tres casos, los que revisamos a continuación.

[...] el hombre necesita la existencia de Dios, tanto para explicar fenómenos desconocidos como para entender la realidad

El ejemplo (14) corresponde a una opinión formulada por un alumno. El uso de la frase nominal definida genérica 'el hombre' otorga el significado evidencial de que la fuente de información es neutral, no necesariamente propia (es decir, su opinión no se expresa como si se basara en la propia experiencia personal) ${ }^{4}$, y que el acceso a la información es universal (una información compartida por todos). Esta estrategia otorga a la opinión apariencia de objetividad y de 'verdad conocida'. 
Este caso, ya comentado en el apartado 3.1., presenta en el enunciado el pronombre 'uno', con el significado de que el juicio se basa en la experiencia personal del hablante, experiencia que, sin embargo, se considera universalizable.

[...] el hombre ha ido descubriendo cosas, pero hay cosas que uno no puede descubrir

El ejemplo (16) presenta nuevamente una alternancia entre dos formas, aunque en esta ocasión el juego se da entre una frase nominal genérica y el pronombre 'uno'. La primera parte del enunciado, expresada con la frase 'el hombre' indica el 'descubrimiento de cosas por el hombre' como una verdad conocida (fuente de información neutral, acceso a la información universal); sin embargo, al momento de manifestar el hablante su opinión sobre la posibilidad de 'descubrirlo todo', prefiere señalar que se basa en su propia experiencia o creencias, aunque se trata de una información que igualmente considera compartida con sus interlocutores, lo que indica con el uso de 'uno'.

\subsubsection{Clase 5 (133 intervenciones)}

La clase 5, cuyo tópico de discusión es el aborto, presenta un caso, el que se puede ver en el ejemplo (17).

[...] uno sabe perfectamente que la mayoría de esos niños [los que van a hogares de adopción] se van a hacer delincuentes

Este enunciado corresponde a la intervención de uno de los estudiantes. La opinión que expresa hace uso del pronombre 'uno', con lo que señala que su comentario se basa en sus propias experiencias o creencias, pero que esta opinión es seguramente compartida por sus interlocutores. Si hacemos el ejercicio de alternar el pronombre ‘uno' por ‘yo' ('yo sé perfectamente...') o por una frase nominal genérica ('la gente sabe perfectamente...'), podemos ver las ventajas argumentativas ya comentadas en el ejemplo (12).

\section{Factores que favorecen la presencia de evidenciales en las clases}

La cantidad total de estrategias evidenciales halladas en el análisis de los diálogos argumentativos es de nueve. Uno de ellos corresponde al uso del pretérito imperfecto con valor de información consabida (ejemplo (7)), mientras que los ocho restantes se relacionan con la elección dentro del paradigma de 'uno'. 
La distribución de los evidenciales en relación con las clases no es equitativa y parece haber varios factores que pueden incidir en su aparición.

\subsection{Cantidad de intervenciones}

El factor más básico que se puede proponer que influye en la frecuencia de aparición de evidenciales es la cantidad de intervenciones de estudiantes en los diálogos producidos durante las clases. En principio, esperaríamos encontrar una relación directa entre número de intervenciones y evidenciales. El Gráfico 1 nos muestra cómo es esta relación.

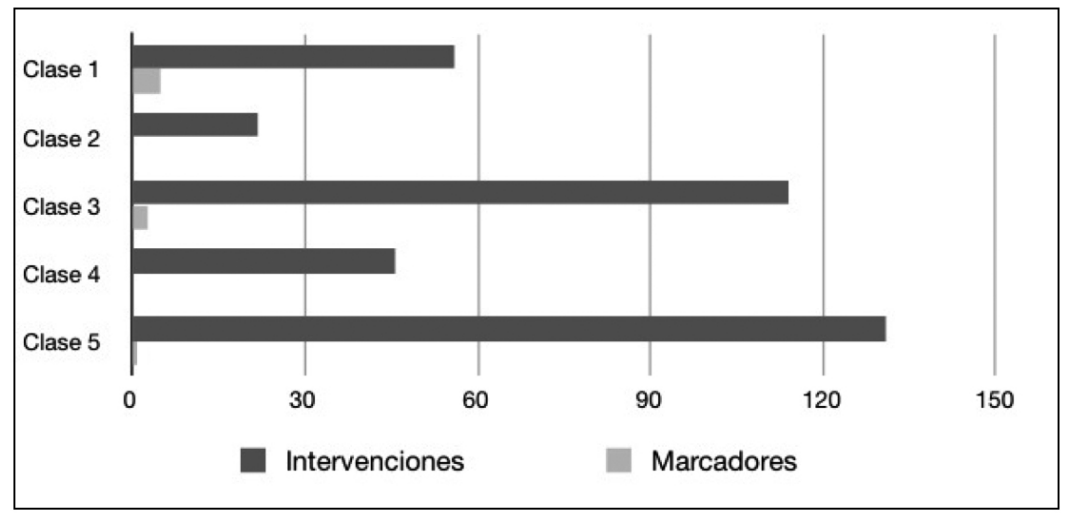

Gráfico 1. Cantidad de marcadores evidenciales y número total de intervenciones por clase.

Como se puede observar en este Gráfico, las dos clases con menor cantidad de intervenciones (la 2 (22) y la 4 (46)) no muestran estrategias evidenciales, por lo que la cantidad de turnos que se dé en la discusión se podría postular en principio como un factor influyente; sin embargo, los datos restantes muestran que no hay realmente una relación directa entre número de intervenciones y cantidad de evidenciales. De hecho, la clase con más intervenciones, la número 5 , presenta solo una estrategia, mientras que la que muestra más cantidad de ellas es la tercera con menos intervenciones, solamente 56 .

Otro punto que este Gráfico ilustra con claridad es la escasa presencia general de estrategias evidenciales, los que en la clase con mayor abundancia de estas, la número 1 , solo alcanzan a aparecer en menos de un $10 \%$ del total de intervenciones. 


\subsection{Temas de discusión}

Un segundo aspecto que se puede postular que influye en la ocurrencia de estrategias evidenciales es el tópico de discusión. La clase que presenta mayor cantidad de estas es la que gira en torno al tema de la aplicación de la jornada escolar completa (clase 1); es decir, un tema de alto interés para los alumnos y uno del que pueden fácilmente entregar experiencias propias. En el otro extremo, encontramos la clase 4, que no presenta estrategias de expresión de la evidencialidad. Esta clase presentó una discusión sobre un tema propuesto por el profesor: la clonación. Resulta claro que este tópico es menos cercano a los estudiantes, lo que puede influir en la ausencia total de estrategias.

Existen, sin embargo, dos clases que parecen contradecir esta tendencia. Por una parte, la clase 2 , cuyo tema de discusión es la conveniencia de que los estudiantes adhieran a movilizaciones estudiantiles. Este tema se asemeja al de la clase 1, en cuanto ambos se relacionan con circunstancias muy cercanas a los estudiantes; aun así, la clase 2 no ofrece estrategias evidenciales (aunque, como discutimos en 3.2., esta clase solamente cuenta con 22 intervenciones, lo que puede ser un factor importante). Por otra parte, podemos observar la clase 3, cuyo tema es la existencia de Dios y la necesidad de este para el hombre. Este tópico, al igual que el de la clase 4, fue propuesto por el profesor y, sin embargo, la clase 3 es la que muestra la segunda mayor cantidad de estrategias evidenciales: 4 en total.

Parece, entonces, que el tópico de discusión de la clase, si bien puede influir, no es un factor claro en la determinación de la cantidad de estrategias evidenciales.

\subsection{Grado de estructuración de la clase}

Un último aspecto que se puede proponer como influyente para la aparición de evidenciales es el grado de estructuración de la clase. Del total de cinco clases analizadas, tres de ellas (las clases 4,5 y 6) corresponden a debates altamente estructurados, en los que dos grupos de estudiantes presentan puntos de vista opuestos sobre un mismo tema planteado con anterioridad por el profesor y sobre el cual han tenido tiempo para buscar referencias bibliográficas, una (la clase 1) corresponde a una clase expositiva en la que los alumnos participan libremente y, finalmente, la restante (clase 2) es una discusión espontánea entre los estudiantes, a la manera de un consejo de curso.

Lo primero que llama la atención es que de las tres clases correspondientes a debates fuertemente estructurados, dos de ellas presentan bajo número de estrategias evidenciales: la 5 solamente una, mientras que la 4 no muestra ninguna. Esto no quiere decir, sin embargo, que en los debates formales los estudiantes no hagan referencia a las fuentes de información en que basan sus aseveraciones. Lo que parece suceder es que en estos casos los hablantes prefieren 
utilizar medios léxicos o frases específicas para señalar sus fuentes, como puede apreciarse en los ejemplos de (18), tomados de estas clases.

(18) a. [...] el hombre, por su capacidad de razonamiento, puede descubrir. Eso es lo que dice Kant

b. $\quad[\ldots]$ en la filosofía hay un principio que habla sobre el principio de ser excluido que dice: «la negación de un ente conforma la de un universo posible»

c. $\quad[\ldots]$ como dice la tesis del conocimiento científico: «para también mejorar el [?] hasta ahora y, por último, mejorar lo que es [?] y así posteriormente salvar más vidas que ahora no se pueden salvar»

d. [lo] primero que escuché en tus palabras, nombraste lo que es la bioética

e. $\quad[. .$.$] podríamos decir, etimológicamente, que el aborto tiene una, la «a»,$ que significa 'eliminación', mientras que «borto» significa 'nacimiento'; por lo tanto, significaría 'eliminación del nacimiento'

En estos ejemplos, podemos ver que las fuentes que los alumnos citan son variadas: autoridades como 'Kant' (18a), principios teóricos más o menos específicos como 'la filosofía' (18b) o ‘la tesis del conocimiento científico' (18c), el discurso anterior de los interlocutores (18d) o, incluso, la etimología de términos en cuestión (18e).

Una cuestión interesante que sucede al exponer las fuentes de información mediante recursos léxicos, como en los casos de (18), es la posibilidad de refutación por el interlocutor, como se observa en el ejemplo (19), tomado de la clase 5. En este caso, se presenta como fuente de información 'la comprobación científica', fuente de alto prestigio, la que, sin embargo, no es aceptada como válida por el otro participante en el debate.

Está científicamente comprobado que el ser que está dentro del útero de la mujer es solamente un conglomerado de células hasta que nace.

Bueno, no sé qué fuentes has visto tú...

Este tipo de refutaciones no se presenta, sin embargo, cuando el hablante hace uso de estrategias evidenciales, lo que podría conducir a pensar que el uso de estas es un mejor recurso en términos de persuasión.

La clase que presenta mayor cantidad de evidenciales (clase 1) corresponde a una exposición del profesor con participación libre y constante de los alumnos. El contraste observado parece 
sugerir que una discusión menos estructurada favorece la presencia de evidenciales expresados por medios gramaticales, mientras que una interacción con mayor estructura formal favorece la presentación de la fuente de información por medios léxicos.

Esta tendencia, sin embargo, puede ser puesta en duda al observar que la interacción menos estructurada de todas, la discusión de la clase 2, no presenta ninguna estrategia evidencial, aunque nuevamente esto puede explicarse por la baja cantidad de intervenciones totales en esta clase.

\section{DISCUSIÓN Y CONCLUSIONES}

Tras el análisis realizado, podemos concluir que los estudiantes de enseñanza media cuyas interacciones verbales componen nuestro corpus sí hacen uso de estrategias gramaticales evidenciales para señalar la fuente de información en la argumentación oral en clases. Este uso, sin embargo, es bastante escaso en relación con el número de intervenciones totales presentadas en estas clases: nueve evidenciales en 373 intervenciones.

De las estrategias evidenciales descritas con anterioridad para el español solo encontramos la ocurrencia de un caso en las clases: el uso del pretérito imperfecto con valor de información consabida. Creemos haber descubierto, eso sí, un evidencial no contemplado en los estudios anteriores sobre el tema: el uso del pronombre de tercera persona 'uno', en alternancia paradigmática con el pronombre de primera persona 'yo' y con una frase nominal de interpretación genérica que incluya al hablante como 'el ser humano'. Cada una de estas formas posee un valor evidencial diferente, producto de la interacción de los ejes 'fuente de información' y 'acceso a la información', propuestos en Bermúdez (2005a). 'Uno' y su paradigma son los evidenciales con mayor presencia en las argumentaciones orales analizadas, con un total de ocho casos.

Finalmente, de entre los factores que se puede postular que influyen en la mayor presencia de evidenciales en el diálogo, nos parece que la cercanía del hablante con respecto al tema (lo que le otorga la posibilidad de basar los argumentos en sus experiencias o creencias) y un bajo grado de estructuración de la discusión favorecen la aparición de estrategias evidenciales.

En consecuencia, nos parece posible proponer que el carácter argumentativo propio de actividades orales como las discusiones en clase estimula al hablante a recurrir a posibilidades lingüísticas que le permiten aumentar el peso persuasivo de su intervención, posibilidades entre las que se encuentran las estrategias evidenciales revisadas. Sin embargo, dado el carácter exploratorio de la presente investigación, para poder afirmar esto con certeza es imprescindible contrastar los hallazgos aquí descritos con otras que se centren en el análisis de otros tipos textuales o prácticas discursivas. 


\section{REFERENCIAS BIBLIOGRÁFICAS}

Aikhenvald, A. (2004). Evidentiality. Oxford: Oxford University Press.

Bermúdez, F. (2002). La estructura evidencial del castellano: Elevación de sujeto y gramaticalización, comunicación presentada al XV Skandinaviske Romanistkongress, Oslo, Noruega.

Bermúdez, F. (2005a). Evidencialidad: La codificación lingüística del punto de vista. Tesis doctoral, Universidad de Estocolmo, Estocolmo, Suecia.

Bermúdez, F. (2005b). Los tiempos verbales como marcadores evidenciales. El caso del pretérito perfecto compuesto. Estudios Filológicos, 40, 165-188.

Chafe, W. \& Nichols, W. (Eds.) (1986). Evidentiality: The linguistic coding of epistemology. Norwood, NJ: Ablex.

De Haan, F. (1999). Evidentiality and epistemic modality: Setting boundaries. Southwest Journal of Linguistics, 18, 83-101.

DeLancey, S. (2001). The mirative and evidentiality. Journal of Pragmatics, 33, 369-382.

Demonte, V. \& Fernández, O. (2005). Features in comp and syntactic variation: The case of '(de) queísmo' in Spanish. Lingua, 115(8), 1063-1082.

Dixon, R. (1988). A grammar of Boumaa Fijian. Chicago: University of Chicago Press.

Lazard, G. (1999). Mirativity, evidentiality, mediativity, or other? Linguistic Typology, 3, 91 109.

Lazard, G. (2001). On the grammaticalization of evidentiality. Journal of Pragmatics, 33, 359367.

Plungian, V. (2001). The place of evidentiality within the universal grammatical space. Journal of Pragmatics, 33, 349-357.

Rooryck, J. (2001). Evidentials. Parts I, II. Glot International, 5, 125-133.

Schwenter, S. (1999). Evidentiality in Spanish morphosyntax: A reanalysis of '(de)queísmo'. En M. Serrano (Ed.). Estudios de variación sintáctica (pp. 65-87). Madrid: Vervuet-Iberoamericana.

Tournadre, N. (1996). Comparaison des systèmes médiatifs de quatre dialectes Tibétains (Tibétain Central, Ladakhi, Dzongkha et Amdo). En Z. Guentchéva (Ed.). L'énonciation médiatisée (pp. 195-213). París: Peeters.

van Eemeren, F., Houtlosser, P. \& Snoeck Henkemans, F. (2007). Argumentative indicators in discourse. A pragma-dialectical study. Dordrecht: Springer.

Wilett, T. (1988). A cross-linguistic survey of the grammaticization of evidentiality. Studies in Language, 12, 51-97. 


\section{NOTAS}

1 En el apartado 1.1. de este trabajo presentamos algunos ejemplos precisos de los recursos gramaticales utilizados para la expresión del significado evidencial en lenguas como el tariana o el boumaa.

2 Las siguientes son las abreviaturas utilizadas en las glosas: 1,2,3 = primera, segunda, tercera personas gramaticales; CLASIF = clasificador; INFER = información inferida; ART = artículo; $\mathrm{NF}=$ no final; NOVIS = sensorial no visual; PAS = pasado; PASREC = pasado reciente; $\mathrm{PL}$ = plural; PRESUN = información presunta; REP = información reportada; $\mathrm{SG}$ = singular; $\mathrm{TR}$ = transitivo; $\mathrm{vIS}$ = sensorial visual.

3 Este ejemplo se presenta aquí ligeramente alterado para que la expresión sea más clara. La intervención original es citada en (15) y comentada en el apartado 3.2.2.

$4 \quad$ Es importante recordar que lo que está en juego en la expresión del significado evidencial no es si el hablante se basa en su experiencia al admitir su opinión, sino si ha elegido expresarlo de esta manera en su intervención. 